\title{
(3) In vitro transcription of guide RNAs and 5'-triphosphate removal V.12
}

\author{
Mark Dewitt ${ }^{1}$, Julia Wong ${ }^{1}$, Beeke Wienert ${ }^{1}$, Moritz F Schlapansky ${ }^{2}$ \\ ${ }^{1}$ UC BERKELEY-IGI; ${ }^{2}$ ETHZ - ETH Zurich

\section{CornLab}

\section{VERSION 12}

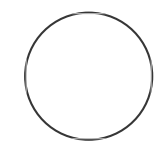

Moritz F Schlapansky

ETHZ - ETH Zurich

\section{ABSTRACT}

sgRNA template assembly, in vitro T7 transcription, and sgRNA column cleanup to remove 5 -triphosphate groups

\section{DOI:}

dx.doi.org/10.17504/protocol s. io. bqjbmuin

\section{External link:}

https://doi.org/10.1371/journa I. pbio.2005840

Protocol Citation: Mark Dewitt, Julia Wong, Beeke Wienert, Moritz F Schlapansky 2020. In vitro transcription of guide RNAs and 5'triphosphate removal. protocols.io

https://dx.doi.org/10.17504/p rotocols. io. bqjbmuinVersion created by Moritz F Schlapansky

\section{MANUSCRIPT CITATION:} Wienert B, Shin J, Zelin E, Pestal K, Corn JE (2018) In vitro-transcribed guide RNAs trigger an innate immune response via the RIG-I pathway. PLoS Biol 16(7): e2005840. doi: $\underline{10.1371 / \text { journal.pbio. } 200584}$ $\underline{0}$ 
License: This is an open access protocol distributed under the terms of the Creative Commons Attribution License, which permits unrestricted use, distribution, and reproduction in any medium, provided the original author and source are credited

Protocol status: Working We use this protocol and it's working

Created: Dec 09, 2020

Last Modified: Dec 09, 2020

PROTOCOL integer ID: 45379

\section{GUIDELINES}

The primers used are: one long, variable oligo that carries the T7 promoter and desired guide sequence; an 82-nt constant oligo that carries the $3^{\prime}$ end of the sgRNA; two short external primers for amplification.

\section{Assembly Oligos:}

T7FwdVar oligo (5'-TAATACGACTCACTATAG--protospacer sequenceGTTTCAGAGCTATGCTGGAAAC-3' )

T7RevLong oligo (5'-

\section{AAAAAAAGCACCGACTCGGTGCCACTTTTTCAAGTTGATAACGGACTAGCCTTATTT CAACTTGCTATGCTGTTTCCAGCATAGCTCTGA-3' )}

\section{Amplification Primers:}

T7FwdAmp primer (5'-TAATACGACTCACTATAG-3')

T7RevAmp primer (5'-AAAAAAAGCACCGACTCGGTGC-3')

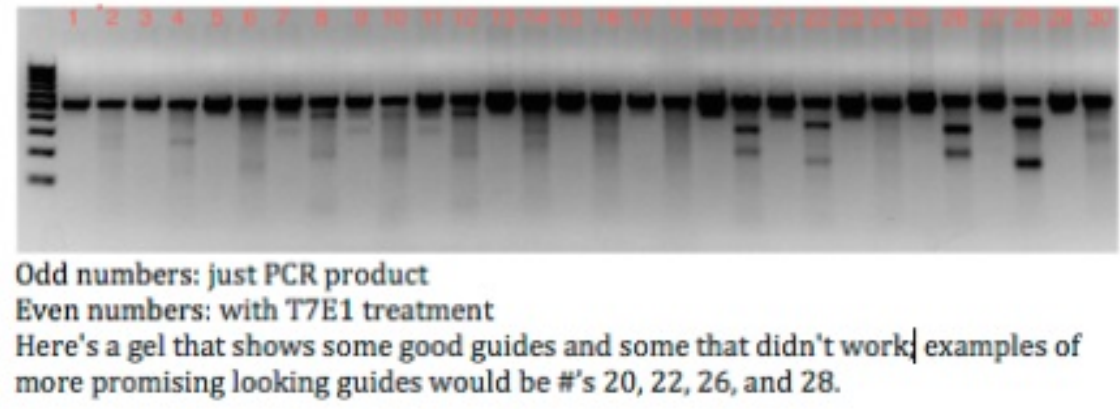

Designing the guide sequence:The $T 7$ transcript starts with the $\mathrm{G}$ before the dashes in the Fwd assembly oligo. If your target calls for a $G$ at the $5^{\prime}$ end of the guide, use the one already included in T7FwdVar design. If your target calls for a different base at the $5^{\prime}$ end, put the whole guide sequence in T7FwdVar; there will be an extra $\mathrm{G}$ on the end that should have minimal effect, unless your guide is quite short.

Making the template: we have used the Phusion-HF DNA polymerase. Although the amplification primers have quite different Tm's, they work together fine. 
MATERIALS

STEP MATERIALS

88 DNase I (RNase-free) - 1,000 units New England Biolabs Catalog \#M0303S

88 HiScribe T7 High Yield RNA Synthesis Kit - 50 rxns New England

Biolabs Catalog \#E2040S

8 Shrimp Alkaline Phosphatase (rSAP) - 500 units New England Biolabs Catalo \#M0371S

\section{Design sgRNA and order PCR oligos.}

Add the desired protospacer sequence to the T7FwdVar oligo and order the oligo from your favorite oligonucleotide supplier. There are many programs available for protospacer design that attempt to optimize on- and/or off-target activity. Which program is most useful depends upon many factors including type of editing, organism being edited, etc. Choice of protospacer design program is beyond the scope of this protocol.

The transcript will start with the bolded $G$ just $5^{\prime}$ of the dashes in the T7FwdVar oligo. T7 RNA polymerase requires a $5^{\prime} G$ for proper transcript initiation. If your protospacer has a $G$ at the $5^{\prime}$ end, you can omit it from the T7FwdVar design to avoid duplication of the $G$. If your protospacer has a $C, T$, or $A$ at the $5^{\prime}$ end, add the whole protospacer sequence to T7FwdVar. In this case, there will be an extra $G$ added to the $5^{\prime}$ end of the protospacer, but literature indicates this will have minimal effect unless your guide is very short.

Primers:

T7FwdVar oligo (5'-TAATACGACTCACTATAG--protospacer sequenceGTTTCAGAGCTATGCTGGAAAC-3' )

T7RevLong oligo (5'-

AAAAAAAGCACCGACTCGGTGCCACTTTTTCAAGTTGATAACGGACTAGCCTTATTTCAACTTGCT ATGCTGTTTCCAGCATAGCTCTGA-3' )

T7FwdAmp primer (5'-TAATACGACTCACTATAG-3')

T7RevAmp primer (5'-AAAAAAAGCACCGACTCGGTGC-3')

\section{Generate in vitro transcription DNA template}

2 For each T7FwdVar oligo you designed, set up the following PCR (total volume should be $20.0 \mu \mathrm{L}$ ). Make sure everything is RNase free and filter tips are used. Furthermore, wipe down everything (in every step of protocol) with RNase Away to ensure no contamination with RNAse.

10.6 $\mu$ I DEPC-treated $\mathrm{H}_{2} \mathrm{O}$

4.0 uL 5x Phusion HF Buffer

$0.4 \mu \mathrm{l} 10 \mathrm{mM}$ dNTPs 


\section{$0.4 \mu \mathrm{l} \mathrm{T7FwdVar}(1 \mu \mathrm{M})$}

$0.4 \mu \mathrm{l}$ T7RevLong $(1 \mu \mathrm{M})$

$2 \mu \mathrm{l} \mathrm{T7FwdAmp}(10 \mu \mathrm{M})$

$2 \mu$ I T7RevAmp $(10 \mu \mathrm{M})$

$0.2 \mu \mathrm{l}$ Phusion HF DNA polymerase $(2 \mathrm{u} / \mu \mathrm{l})$

If making multiple sgRNA templates, prepare a master mix with all components except T7FwdVar. Include a no template control (omit T7FwdVar).

Primers:

T7FwdVar oligo (5'-TAATACGACTCACTATAG--protospacer sequence-

GTTTCAGAGCTATGCTGGAAAC-3' )

T7RevLong oligo (5'-

AAAAAAAGCACCGACTCGGTGCCACTTTTTCAAGTTGATAACGGACTAGCCTTATTTCAACTTGCT

ATGCTGTTTCCAGCATAGCTCTGA-3' )

T7FwdAmp primer (5'-TAATACGACTCACTATAG-3')

T7RevAmp primer (5'-AAAAAAAGCACCGACTCGGTGC-3')

\section{In vitro transcription DNA template PCR}

3 Run PCR:

$98^{\circ} 30 \mathrm{sec}$

$98^{\circ} 10 \mathrm{sec}$

$51^{\circ} 10 \mathrm{sec}$

$72^{\circ} 10 \mathrm{sec}$

$30 x$ steps $2-4$

$72^{\circ} 2$ min

$4^{\circ}$ hold

No PCR cleanup necessary at this point

\section{In vitro $\mathrm{T7}$ transcription}

4 We like to use HiScribe T7 High Yield RNA Synthesis Kit but any T7 RNA synthesis kit should be fine.

Mix the following to make $\mathbf{2 0} \boldsymbol{\mu l}$ total T7 transcription mix

\begin{tabular}{|l|l|}
\hline A & B \\
\hline volume & reagent \\
\hline $2 \mu \mathrm{l}$ & $10 x$ Buffer $1 \mathrm{x}$ \\
\hline $2 \mu \mathrm{l}$ & ATP $(100 \mathrm{mM}), 10 \mathrm{mM}$ \\
\hline
\end{tabular}




\begin{tabular}{|l|l|}
\hline A & B \\
\hline $2 \mu \mathrm{l}$ & GTP $(100 \mathrm{mM}), 10 \mathrm{mM}$ \\
\hline $2 \mu \mathrm{l}$ & CTP $(100 \mathrm{mM}), 10 \mathrm{mM}$ \\
\hline $2 \mu \mathrm{l}$ & \begin{tabular}{l} 
UTP $(100 \mathrm{mM}), 10 \mathrm{mM}$ \\
\hline $8 \mu \mathrm{l}$
\end{tabular} \\
\hline $\begin{array}{l}\text { DNA template from Step 3, (usually } \sim 4 \mu \mathrm{g}, 50 \\
\text { pmol })\end{array}$ \\
\hline $2 \mu \mathrm{l}$ & T7 RNA polymerase mix \\
\hline
\end{tabular}

HiScribe T7 High Yield RNA Synthesis Kit - 50 rxns New England Biolabs Catalog \#E2040

5 Incubate transcription mix for $\sim 18$ hours (over night) at $37^{\circ}$ in a thermocycler

(7) $18: 00: 00$

$37^{\circ} \mathrm{C}$

\section{DNA template removal}

6 Remove DNA template by adding $1 \mu$ l of RNase-free DNase; incubate 15 min at 37C in thermocycler

00:15:00 37C incubation

DNase I (RNase-free) - 1,000 units New England Biolabs Catalog \#M0303S

$37^{\circ} \mathrm{C}$

\section{Removal of $5^{\prime}$-triphosphate groups}

$7 \quad$ T7 in vitro transcription results in RNA carrying a 5'-triphosphate group. This triggers a RIG-Imediated innate immnue response in mammalian cells and can cause cell death, particularly in primary cells. We highly recommend treating your IVT sgRNA with recombinant Shrimp Alkaline Phosphatase (rSAP) before proceeding to the purification step. We found that rSAP treatment must be rigorous to completely remove all 5-PPP groups from your RNA. However, rSAP binds tightly to RNA and NEB recommends to only use the minimal amount needed.

8 Add 3ul of NEB CutSmart Buffer (comes with the rSAP enzyme) Add $4 \mu \mathrm{l}$ of $\mathrm{H} 2 \mathrm{O}$

Add 2ul (2 units) of rSAP

\section{Shrimp Alkaline Phosphatase (rSAP) - 500 units New England Biolabs Catalog \#M0371S}

$9 \quad$ Mix well and incubate at $37 \mathrm{C}$ for $3 \mathrm{~h}$

03:00:00 


\section{8. $37^{\circ} \mathrm{C}$}

\section{sgRNA purification}

10 sgRNAs need to be purfied before transfection. There are different methods one could purify their sgRNAs. We found that while SPRI bead clean-up of RNAs is quick and gives reliable yields (see older versions of protocol), SPRI bead purified sgRNAs still cause an elevated immune response even after Phosphatase treatment. We therefore tested different column purification kits and found that column purification of sgRNAs completely eliminates the immune response after Phosphatase treatment.

We use the Qiagen RNeasy Mini Prep Kit and follow the manufacturer's instructions with the following modifications:

\section{Note}

Yields of from column purification can be low as the sgRNAs are small and most kits are designed to bind longer RNAs. We found the Qiagen RNeasy kit works ok, but others recommend the miRNeasy kit from Qiagen. It might be worth trying a few different kits to optimize your yield.

11 Adjust sgRNA sample to a volume of $100 \mu$ with RNase-free water. Add $350 \mu \mathrm{l} \mathrm{RLT}$ Buffer to the sample

12 Add $250 \mu \mathrm{l} 100 \%$ ethanol and mix well by pipetting.

13 Tranfer the sample $(\sim 700 \mu \mathrm{l})$ to an RNeasy mini spin column; spin for $15 \mathrm{sec}$ at $>8000 \mathrm{~g}$ Then transferr the remainder onto the same column; spin for $15 \mathrm{sec}$

$14 \quad$ Add $500 \mu \mathrm{l}$ RPE Buffer; spin $15 \mathrm{sec}$

Repeated this wash step

15 Moved spin column to a new collection tube and spin for 1 min to dry the membrane completely 
16 Move spin column to an RNAse-free $1.5 \mathrm{ml}$ microfuge tube Add $33 \mu$ l DEPC-treated H2O; spin 1 min

Optional: Repeat the elution to collect any remaining RNA

17 Measure your RNA concentration by Nanodrop or Qubit. You can also check integrity/corrrect size of the sgRNA(s) on the RNA tapestation. Store sgRNAs at $-80 \mathrm{C}$ 